\title{
Dysfunction of fibroblasts of extrarenal origin underlies renal fibrosis and renal anemia in mice
}

\author{
Nariaki Asada, ${ }^{1}$ Masayuki Takase, ${ }^{1}$ Jin Nakamura, ${ }^{1}$ Akiko Oguchi, ${ }^{1}$ Misako Asada, ${ }^{1}$ Norio Suzuki, ${ }^{2}$ \\ Ken-ichi Yamamura, ${ }^{3}$ Narihito Nagoshi, 4,5 Shinsuke Shibata, ${ }^{4}$ Tata Nageswara Rao, 6 \\ Hans Joerg Fehling, ${ }^{6}$ Atsushi Fukatsu, ${ }^{7}$ Naoko Minegishi, 2,8 Toru Kita, ${ }^{9}$ Takeshi Kimura, ${ }^{10}$ \\ Hideyuki Okano, ${ }^{4}$ Masayuki Yamamoto, ${ }^{2}$ and Motoko Yanagita1,7,10,11

\begin{abstract}
${ }^{1}$ Career-Path Promotion Unit for Young Life Scientists, Graduate School of Medicine, Kyoto University, Yoshida-konoe-cho, Sakyo-ku, Kyoto, Japan. 2Department of Medical Biochemistry, Tohoku University Graduate School of Medicine, Seiryo-cho, Aoba-ku, Sendai, Japan.

${ }^{3}$ Division of Developmental Genetics, Institute of Molecular Embryology and Genetics, Kumamoto University, Kurokami, Kumamoto, Japan. ${ }^{4}$ Department of Physiology and 5Department of Orthopedic Surgery, Keio University School of Medicine, Shinanomachi, Shinjuku-ku, Tokyo, Japan. ${ }^{6}$ Institute of Immunology, University of Clinics UIm, Ulm, Germany. 'Department of Artificial Kidneys, Graduate School of Medicine, Kyoto University, Yoshida-konoe-cho, Sakyo-ku, Kyoto, Japan. ${ }^{8}$ Graduate School of Nursing, Miyagi University, Miyagi, Japan. ${ }^{9}$ Kobe City Medical Center General Hospital, Kobe, Japan. ${ }^{10}$ Department of Cardiovascular Medicine and ${ }^{11 H a k u b i}$ Project, Graduate School of Medicine, Kyoto University, Yoshida-konoe-cho, Sakyo-ku, Kyoto, Japan.
\end{abstract}

\begin{abstract}
In chronic kidney disease, fibroblast dysfunction causes renal fibrosis and renal anemia. Renal fibrosis is mediated by the accumulation of myofibroblasts, whereas renal anemia is mediated by the reduced production of fibroblast-derived erythropoietin, a hormone that stimulates erythropoiesis. Despite their importance in chronic kidney disease, the origin and regulatory mechanism of fibroblasts remain unclear. Here, we have demonstrated that the majority of erythropoietin-producing fibroblasts in the healthy kidney originate from myelin protein zero-Cre $(\mathrm{PO}-\mathrm{Cre})$ lineage-labeled extrarenal cells, which enter the embryonic kidney at $\mathrm{E} 13.5$. In the diseased kidney, $\mathrm{PO}$-Cre lineage-labeled fibroblasts, but not fibroblasts derived from injured tubular epithelial cells through epithelial-mesenchymal transition, transdifferentiated into myofibroblasts and predominantly contributed to fibrosis, with concomitant loss of erythropoietin production. We further demonstrated that attenuated erythropoietin production in transdifferentiated myofibroblasts was restored by the administration of neuroprotective agents, such as dexamethasone and neurotrophins. Moreover, the in vivo administration of tamoxifen, a selective estrogen receptor modulator, restored attenuated erythropoietin production as well as fibrosis in a mouse model of kidney fibrosis. These findings reveal the pathophysiological roles of P0-Cre lineage-labeled fibroblasts in the kidney and clarify the link between renal fibrosis and renal anemia.
\end{abstract}

\section{Introduction}

Erythropoietin (EPO) is a hormone that is essential for the production of red blood cells (1). EPO is mainly produced in the kidney after birth, and its production is severely reduced in patients with chronic kidney disease (CKD) (2) with renal anemia. The cloning of the EPO gene and the production of recombinant human EPO revolutionized the management of patients with $\mathrm{CKD}$, providing an opportunity for safe long-term anemia correction, which improves cognitive function, quality of life, exercise capacity, and cardiac function (3). Nowadays, an increasing number of patients with CKD receive erythropoiesis-stimulating agents (ESAs), and annual US prescription sales for ESAs reached 10 billion dollars in 2006. In addition to the hematopoietic function of EPO, increasing evidence suggests that EPO plays a role as a tissue-protective factor in various nonhematopoietic tissues (4), raising the possibility that it will be prescribed for various types of tissue injuries in the near future.

Studies using in situ hybridization and the transgenic mice approach indicate that EPO is mainly produced by the interstitial

Authorship note: Nariaki Asada and Masayuki Takase contributed equally to this work.

Conflict of interest: The authors have declared that no conflict of interest exists. Citation for this article: J Clin Invest. 2011;121(10):3981-3990. doi:10.1172/JCI57301. fibroblasts in the deep cortex and the outer medulla in the kidney $(5,6)$. Although the essential role of EPO has been well recognized, the identity and developmental origin of EPO-producing cells in the kidneys remain unknown. This has hindered both the generation of EPO-producing cells from stem cells as well as the clarification of the molecular mechanisms underlying the defective production of EPO in patients with CKD - EPO-producing cells may lose their ability to produce EPO after injury, or, alternatively, EPO-producing cells may be lost during CKD.

Fibroblasts are interstitial mesenchymes that structurally support epithelia by producing ECM. Recently, the tissue-resident fibroblasts have attracted considerable attention because of their prominent roles in fibrosis, endocrine, organogenesis, and tumorigenesis (7). In chronic kidney injury, sustained inflammation accompanies the proliferation of interstitial fibroblasts and myofibroblasts (8), leading to renal fibrosis, which is the final common pathway for all CKD and eventually leads to renal failure (9). The origin of scar-producing myofibroblasts is of prime importance in understanding the mechanisms of renal fibrosis, which remain controversial. The contribution of bone marrow-derived fibrocytes (10) and the epithelial-mesenchymal transition (EMT) of injured tubular epithelial cells (11) has been proposed in some studies, but other groups have reported conflicting results (12-16). 
A
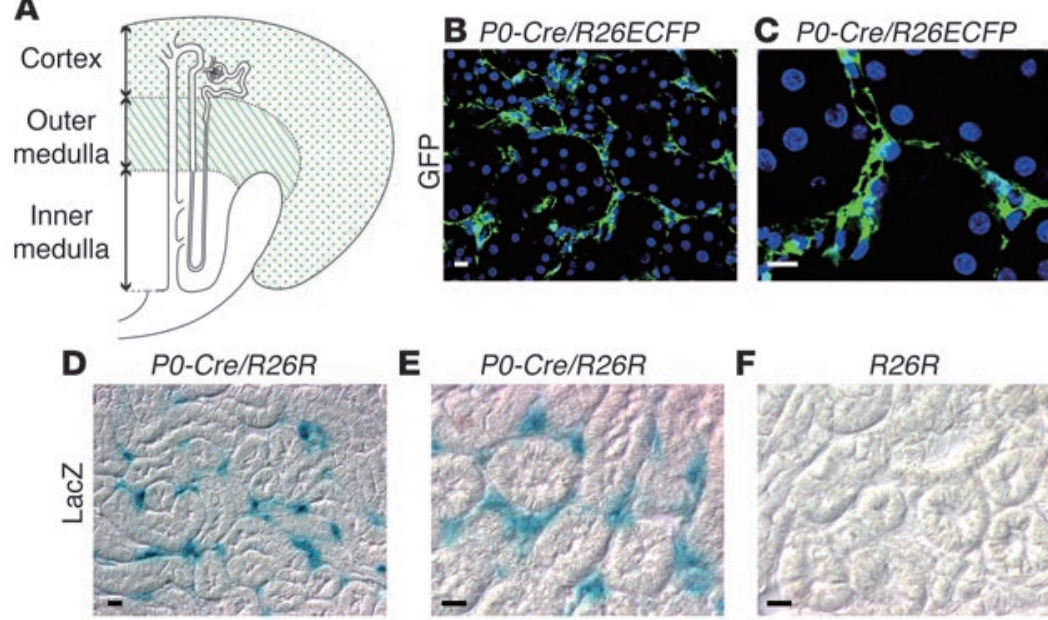

E
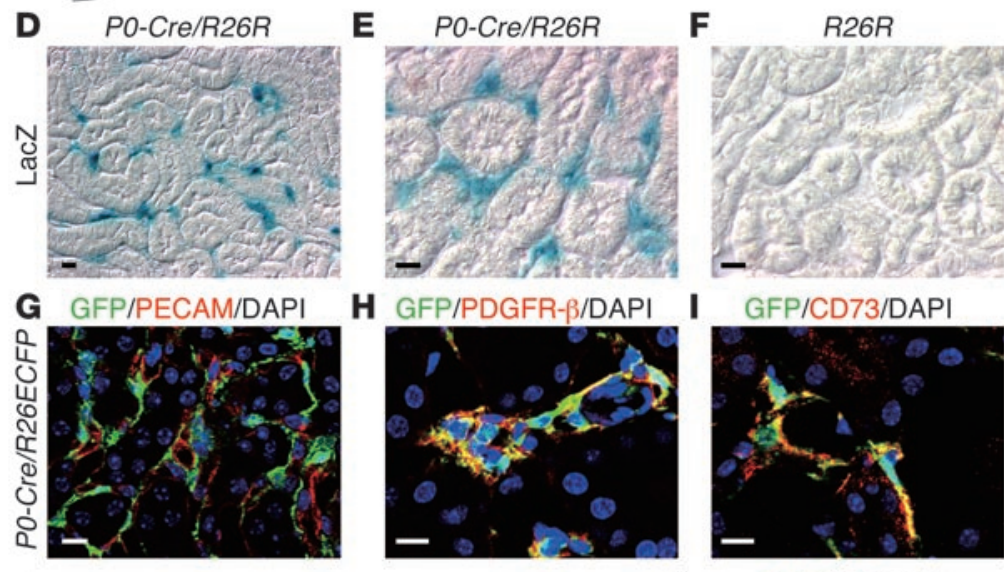

J

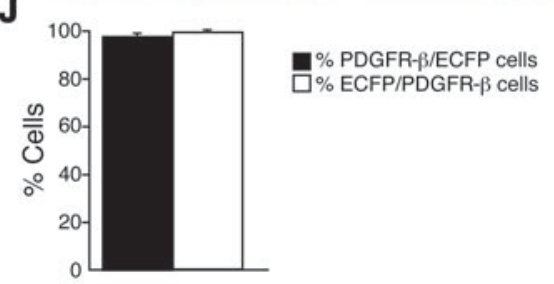

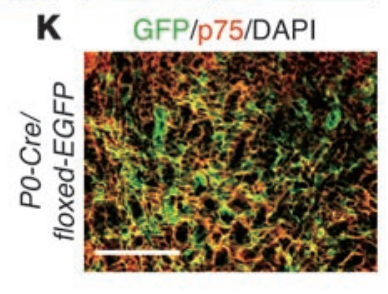

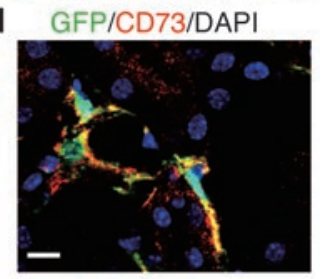

\section{Figure 1}

Most of the fibroblasts in the kidney arise from $\mathrm{PO}-\mathrm{Cre}-$ expressing precursors. (A) Schematic drawing of the kidney, showing the localization of $\mathrm{PO}$-Cre fate-mapped cells in the cortex and outer medulla. (B and C) ECFP+ cells were detected in the interstitium of the kidneys of $P 0-C r e / R 26 E C F P$ mice. A higher-magnification view is shown in C. (D-F) LacZ staining of $P 0-C r e / R 26 R$ kidney. (D and E) Note the similar distribution of $\mathrm{LacZ}^{+}$cells in P0-Cre/R26R kidneys with $\mathrm{ECFP}^{+}$cells in P0-Cre/R26ECFP mice. A higher-magnification view is shown in $E$. $(F)$ No LacZ $^{+}$ cells were observed in the kidneys of $R 26 R$ mice without the $P 0$-Cre allele. (G-I) Double immunostaining of ECFP ${ }^{+}$cells in the P0-Cre/R26ECFP kidney. (G) Most, if not all, of the ECFP+ ${ }^{+}$cells were negative for PECAM. (H and I) ECFP+ cells $(\mathbf{H})$ in the kidney were positive for PDGFR- $\beta$, the fibroblast marker, whereas $\mathrm{ECFP}^{+}$cells (I) in the cortex were also positive for $C D 73 / 5^{\prime} \mathrm{NT}$, the marker for cortical fibroblasts. (J) Graph illustrating the proportion of PDGFR- $\beta^{+}$ interstitial cells in the cortex and outer medulla coexpressing ECFP (\% ECFP/PDGFR- $\beta$ cells) and the proportion of ECFP+ interstitial cells coexpressing PDGFR- $\beta$ (\% PDGFR- $\beta$ /ECFP cells). (K) In neonatal kidneys of PO-Cre/floxed-EGFP mice most of the $\mathrm{EGFP}^{+}$cells were also positive for $\mathrm{p} 75$, a neural crest marker. Scale bars: $10 \mu \mathrm{m}$ (B-I); $100 \mu \mathrm{m}(\mathbf{K})$.
Myelin protein zero (P0) is expressed in migrating neural crest cells in the early embryonic stages as well as in Schwann cells, which also originate from neural crest, in later stages (17). In P0-Cre mice, the activation of the $P O$ promoter induces the Cre-mediated recombination of reporter alleles, permanently tagging the cells arising from $\mathrm{PO}$-Cre-expressing precursors with the expression of indicator genes.

Here, we demonstrate the occurrence of $\mathrm{PO}$-Cre lineage-labeled fibroblasts in the kidney interstitium and their 2 different characteristics - they produce EPO, which possesses hematopoietic and tissue-protective functions in healthy kidney, and they transdifferentiate into scar-producing myofibroblasts and lose their EPO-producing activity after kidney injury, leading to renal fibrosis and renal anemia. We further demonstrate that the attenuated EPO production in scar-producing myofibroblasts can be restored through the administration of neuroprotective reagents, such as low-dose dexamethasone and neurotrophins. A similar effect was also obtained by the administration of a renoprotective reagent HGF. Moreover, the in vivo administration of tamoxifen, a selective estrogen receptor modulator (SERM), restored attenuated EPO production as well as fibrosis in a mouse model of kidney fibrosis.

These findings provide insights that we believe to be novel into the mechanisms of renal fibrosis and renal anemia in CKD and provide clues that assist in the development of therapeutic approaches to treat these disorders.

\section{Results}

Most fibroblasts in the kidney arise from P0-Cre-expressing precursors. P0-Cre mice were mated to 4 indicator mouse lines: floxed EGFP (18), R26R (19), R26ECFP (20), and R26tdRFP mice (21). Immunostaining of the kidneys of $P 0$-Cre/R26ECFP mice using anti-GFP antibody revealed numerous enhanced cyan fluorescent protein-positive $\left(\mathrm{ECFP}^{+}\right)$cells in the cortex and outer medulla (Figure 1, A-C). The kidneys of R26ECFP mice without the P0-Cre allele contained no $\mathrm{ECFP}^{+}$cells, confirming the specificity of staining (data not shown). LacZ staining in P0-Cre/R26R kidney also revealed the same results (Figure 1, D-F). These P0-Cre fatemapped cells are located in the interstitium, the space between the tubules and nutritive peritubular capillaries, exhibiting a unique stellar shape with numerous projections.

The interstitium of the healthy adult kidney is composed of heterogeneous cells, including macrophages, dendritic cells, and peritubular capillaries (9). Most, if not all, $\mathrm{P0}$-Cre fate-mapped cells in the kidney did not express the macrophage marker CD11b (Supplemental Figure 1A; supplemental material available online with this article; doi:10.1172/JCI57301DS1), F4/80 (Supplemental Figure 1B), the dendritic cell marker major histocompatibility complex class II (Supplemental Figure 1C and Supplemental Figure 7), or the common leukocyte antigen CD45 (Supplemental Figure 7). Although a previous report indicated that endothelial cells in certain tissues are of neural crest origin (22), $\mathrm{ECFP}^{+}$cells in the kidney of P0-Cre/R26ECFP mice did not express PECAM/ 
A GFP

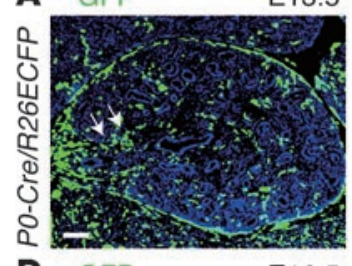

D GFP

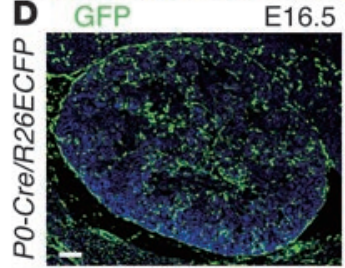

G GFP/Six2

E16.5

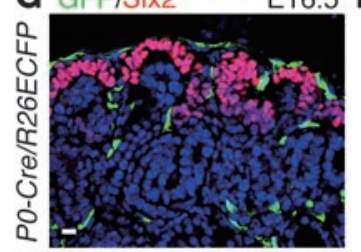

E13.5
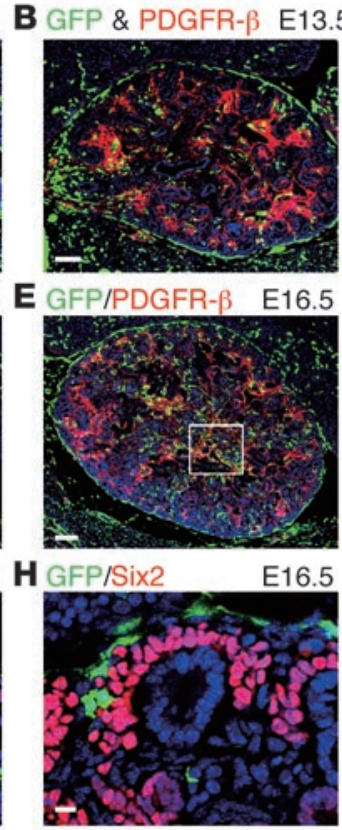

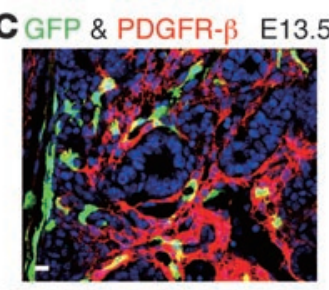

F GFPIPDGFR- $\beta \quad E 16.5$

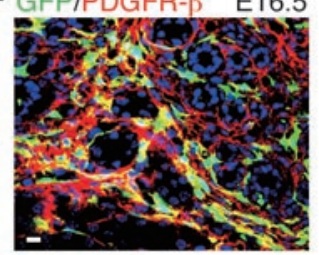

I GFP/TUJI

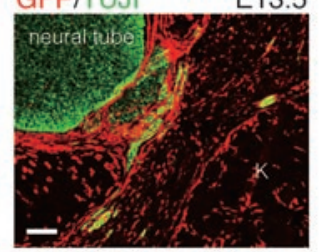

Figure 2

$P 0$-Cre lineage-labeled cells in the developing kidney. (A-C) $\mathrm{ECFP}^{+}$cells were first observed in the kidneys of PO-Cre/R26ECFP mice at E13.5. ECFP+ cells were predominantly located along the outer capsule and the ureter (arrows) of the kidney. (D-F) The number of ECFP+ cells in the kidneys of $P O-C r e /$ $R 26 E C F P$ mice increased at E16.5, and $(F)$ the cells began to express PDGFR- $\beta$. The boxed region in $\mathbf{E}$ is shown at higher magnification in $\mathbf{F}$. ( $\mathbf{G}$ and $\mathbf{H}$ ) $\mathrm{ECFP}+$ cells populating the cortex appeared to surround the territory of Six2+ renal progenitor cells. (I) $\mathrm{ECFP}^{+}$cells in the kidney were negative for class III $\beta$-tubulin. K, kidney. Scale bars: $100 \mu \mathrm{m}$ (A, B, D, E, and I); $10 \mu \mathrm{m}$ (C and F-H).
CD31, a marker for endothelial cells (Figure 1G and Supplemental Figure 7). Very few $\mathrm{ECFP}^{+}$cells expressed tyrosine hydroxylase, a marker for sympathetic nerve fibers, whereas most $\mathrm{ECFP}^{+}$cells did not (Supplemental Figure 2). Instead, $\mathrm{ECFP}^{+}$cells expressed PDGFR- $\beta$, a marker for interstitial fibroblasts in the kidney (refs. 9, 23, and Figure 1H). ECFP ${ }^{+}$cells in the cortex expressed CD73/ ecto-5'-nucleotidase (CD73/5'NT), a marker for cortical fibroblasts (ref. 9 and Figure 1I). More than $98 \%$ of $\mathrm{ECFP}^{+}$interstitial cells expressed PDGFR- $\beta$, whereas more than $99 \%$ of PDGFR- $\beta^{+}$ cells in the interstitium of the cortex and outer medulla expressed ECFP (Figure 1J). Furthermore, $\mathrm{EGFP}^{+}$cells in the neonatal kidneys of $P 0$-Cre/floxed-EGFP mice expressed $\mathrm{p} 75$, a marker for the neural crest (ref. 24 and Figure $1 \mathrm{~K}$ ). These results indicate that most, if not all, of the kidney fibroblasts in the cortex and outer medulla are fate-mapped with $\mathrm{PO}$-Cre.

PO-Cre lineage-labeled cells enter the embryonic kidney during nephrogenesis. To investigate the route and stage of migration of $\mathrm{PO}$-Cre lineage-labeled cells into the kidney, we analyzed P0-Cre/R26ECFP embryos serially from E11.5, when nephrogenesis is initiated, to the neonatal stage. $\mathrm{ECFP}^{+}$cells were first detected from E13.5 onward in metanephric kidneys. These cells were predominantly located along the outer capsule and the ureter of the kidney (Figure 2, A-C), which is possibly indicative of their routes of migration. The number of $\mathrm{ECFP}^{+}$cells in the metanephric kidney dramatically increased in later stages of development, and the $\mathrm{ECFP}^{+}$cells began to express PDGFR- $\beta$ (Figure 2, D-F).

These $\mathrm{ECFP}^{+}$cells populating the cortex appeared to avoid the territory of the Six $2^{+}$renal progenitor cells, which give rise to all nephron segments in the kidney (ref. 25 and Figure 2, G and H). Furthermore, these $\mathrm{ECFP}^{+}$cells did not express class III $\beta$-tubulin, a neuron-specific marker (Figure 2I). Importantly, immunostaining of the P0-Cre embryo failed to identify any $\mathrm{Cre}^{+}$cells in the kidneys throughout development (Supplemental Figure 3, C-E), whereas it detected the expression of Cre in the migrating neural crest and the dorsal root ganglion (Supplemental Figure 3, A and $\mathrm{B})$. Immunostaining of $\mathrm{P} 0$ protein also failed to detect any $\mathrm{P} 0$ protein in the kidneys throughout development (Supplemental Figure 4, A-C). We further demonstrated that the expression of $P 0$ and Cre mRNA was almost undetectable in embryonic kidneys (Supplemental Figure 8), whereas the number of P0-Cre-labeled cells increased substantially during development. Thus, it is highly unlikely that the ectopic expression of Cre in the kidney accounts for $\mathrm{ECFP}^{+}$cells in the kidney.

PO-Cre lineage-labeled fibroblasts produce EPO. Renal EPO-producing cells are thought to be peritubular interstitial cells that exhibit a stellar shape with projections and express neuronal markers (6). Given these features of EPO-producing cells, we examined whether $\mathrm{EPO}$-producing cells arise from $\mathrm{P} 0$-Cre-expressing precursors. We used Epo-GFP mice, a BAC transgenic mouse line that expresses GFP as a reporter under the control of a $180-\mathrm{kb}$ mouse Epo gene locus, and identified the EPO-producing population in the kidney (6).

GFP expression in the kidney of Epo-GFP mice was detected in the interstitium and was colocalized with the fibroblast markers CD73 $/ 5^{\prime} \mathrm{NT}$ and PDGFR- $\beta$ (Figure $3, \mathrm{~A}-\mathrm{C}$ ). Analysis of the kidneys of P0-Cre/R26R/Epo-GFP mice revealed that most of the $\mathrm{GFP}^{+}$cells were positive for LacZ (Figure 3, D and E), supporting the idea that $\mathrm{EPO}$-producing cells originate from the $\mathrm{PO}$-Cre-expressing precursors. We further analyzed the kidneys of P0-Cre/R26tdRFP/EPo-GFP mice and found that more than $75 \%$ of $\mathrm{GFP}^{+}$cells were also positive for tandem-dimer red fluorescent protein (tdRFP) (Figure 3F).

EPO expression in $\mathrm{PO}$-Cre fate-mapped cells was further examined using $\mathrm{EGFP}^{+}$and $\mathrm{EGFP}^{-}$cells FACS-purified from kidneys of adult PO-Cre/floxed-EGFP mice. PCR analysis of these populations revealed the expression of EPO and p75 in $\mathrm{EGFP}^{+}$cells but not in the EGFP- population (Figure $3 \mathrm{G}$ ). Sorting $\mathrm{p}^{+} 5^{+}$cells in the kidneys of P0-Cre/R26ECFP mice at 2 weeks of age also revealed higher expression of $\mathrm{p} 75, \mathrm{EPO}$, and ECFP in $\mathrm{p}^{7} 5^{+}$cells than in $\mathrm{p}^{75^{-}}$cells (Figure $3 \mathrm{H}$ ).

$\mathrm{PO}$-Cre lineage-labeled fibroblasts transdifferentiate into myofibroblasts and contribute to fibrosis. To investigate the response of PO-Cre lineage-labeled fibroblasts to injury, we performed unilateral ureteral obstruction (UUO), a well-established model for renal fibrosis 

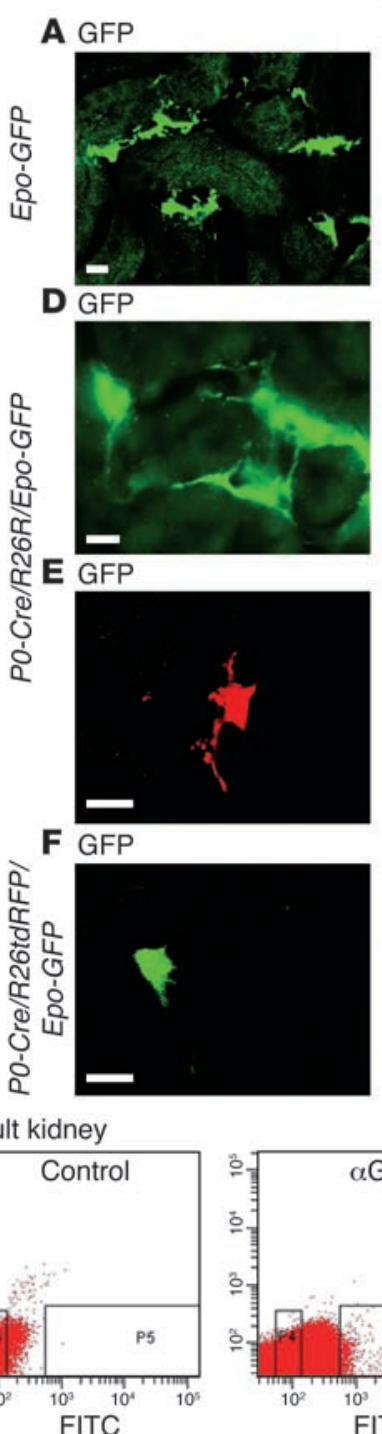

H Neonatal kidney

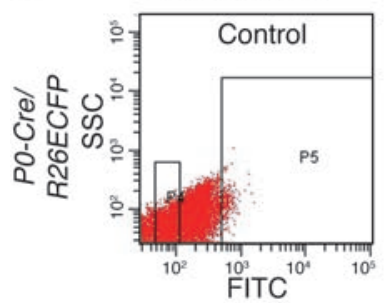

G

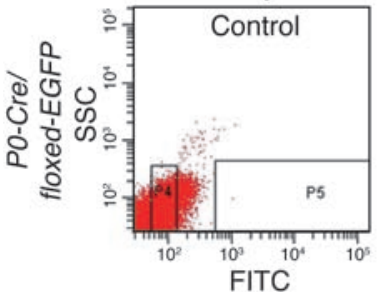

B

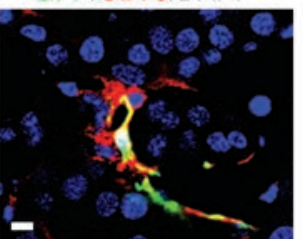

LacZ

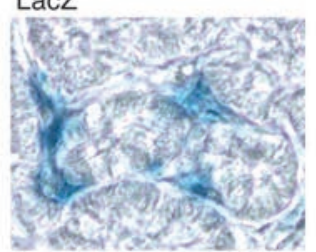

LacZ immunostaining

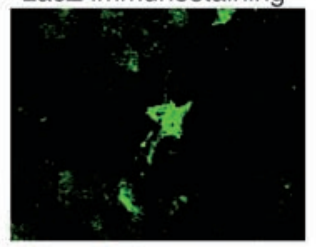

tdRFP

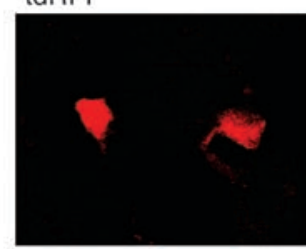

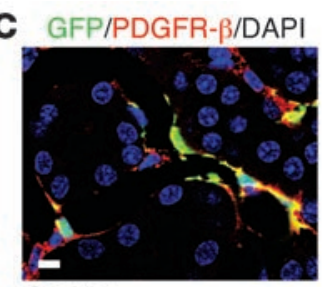

Overlay

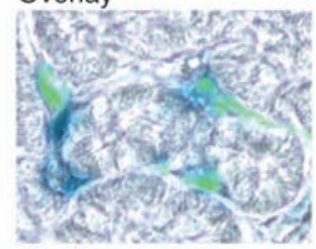

Overlay

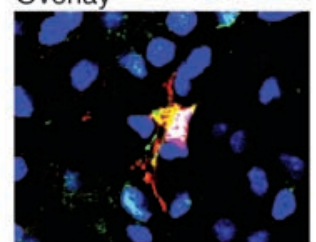

Overlay

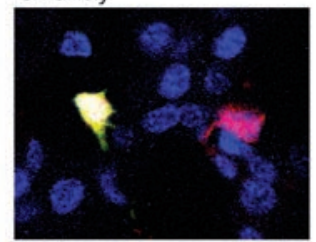

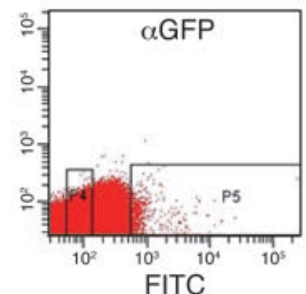

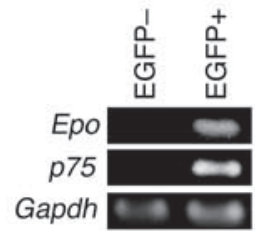

\section{Figure 3}

PO-Cre lineage-labeled fibroblasts produce EPO. (A-C) Stellate-shaped GFP+ cells with projections were detected (A) in the interstitium of the kidneys of Epo-GFP mice and were positive for (B) CD73 and (C) PDGFR- $\beta$. (D and E) Analysis of the kidney of P0-Cre/R26R/Epo-GFP mice revealed that GFP+ cells were also positive for LacZ. (F) Analysis of the kidney of PO-Cre/R26tdRFP/Epo-GFP mice revealed that most GFP+ cells were also positive for tdRFP. Scale bars: $10 \mu \mathrm{m}$. (G) Sorting EGFP+ cells (P5) out of EGFP- cells (P4) from the kidneys of adult P0-Cre/ floxed-EGFP mice. RT-PCR analysis of these populations revealed high expression of Epo and $p 75$ in EGFP+ cells but not in EGFP- cells. Cells were stained either with or without anti-GFP antibody. (H) Sorting p $75^{+}$cells (P5) out of p75- cells (P4) from the kidneys of PO-Cre/R26ECFP mice at 2 weeks of age. Cells were stained either with or without anti-p75 antibody. Quantitative PCR analysis demonstrated that the expression of $p 75, E p o$, and ECFP was higher in $\mathrm{p} 75^{+}$cells. Expression of $p 75$ was normalized to that of Gapdh and expressed relative to that in $\mathrm{p} 75^{-}$cells. 


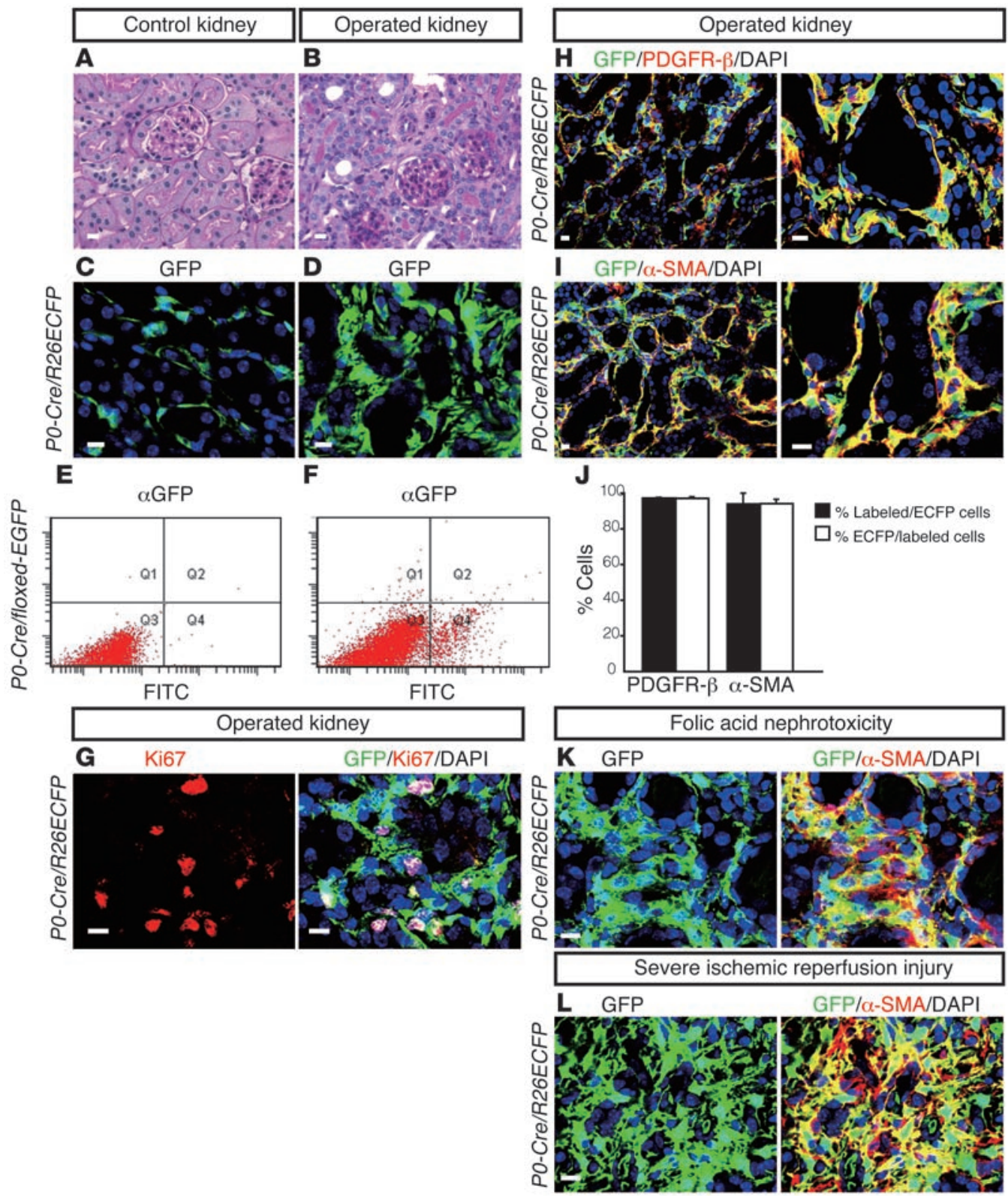

Figure 4

PO-Cre lineage-labeled fibroblasts transdifferentiate into myofibroblasts and contribute to fibrosis. (A and B) Five days after obstruction of the ureter, (B) interstitial fibrosis was prominent in the operated kidney (A) compared with that in the control kidney. (C and $\mathbf{D})$ The number of ECFP+ cells was markedly increased in the operated kidneys of P0-Cre/R26ECFP mice compared with that in the control kidney. (E and F) FACS analysis also confirmed the increase in the number of EGFP+ cells in the operated kidneys of $P 0$-Cre/floxed-EGFP mice. Cells were stained with anti-GFP antibody. (G) ECFP/Ki67 double-positive cells were abundant in the operated kidneys of P0-Cre/R26ECFP mice. (H and I) Most ECFP+ cells were also positive for (H) PDGFR- $\beta$ and (I) $\alpha$-SMA. (J) Graph illustrating the proportion of PDGFR- $\beta^{+}$or $\alpha$-SMA+ interstitial cells coexpressing ECFP (\% labeled/ECFP cells) and the proportion of interstitial ECFP+ cells coexpressing either PDGFR- $\beta$ or $\alpha$-SMA (\% ECFP/labeled cells). ( $\mathbf{K}$ and $\mathbf{L}$ ) The number of ECFP+ cells was also markedly increased in the kidneys of P0-Cre/R26ECFP mice after (K) folic acid nephrotoxicity and $(\mathbf{L})$ severe ischemic reperfusion injury. Most ECFP+ ${ }^{+}$cells were also positive for $\alpha$-SMA. Scale bars: $10 \mu \mathrm{m}$.

(26), using P0-Cre/R26ECFP mice. Five days after UUO, the number of $\mathrm{ECFP}^{+}$cells had markedly increased in the interstitium of the operated kidney, in parallel with the progression of interstitial fibrosis (Figure 4, A-D). FACS analysis also revealed a substantial increase in the number of $\mathrm{EGFP}^{+}$cells in the operated kidney in P0-Cre/floxed-EGFP mice (Figure 4, E and F). It is unlikely that the increase in $\mathrm{ECFP}^{+}$or $\mathrm{EGFP}^{+}$cells is due to the ectopic expression of Cre or P0 protein in the kidney, because neither Cre protein nor $\mathrm{P} 0$ protein was detectable in the operated kidney (Supplemental Figure 3F and Supplemental Figure 4D). We further demonstrated that the expression of $P 0$ and $C r e$ mRNA was almost undetectable in fibrotic kidneys (Supplemental Figure 8), whereas the number of $\mathrm{PO}$-Cre-labeled cells increased substantially during kidney fibrosis. Immunostaining of the operated kidney revealed the presence 
A
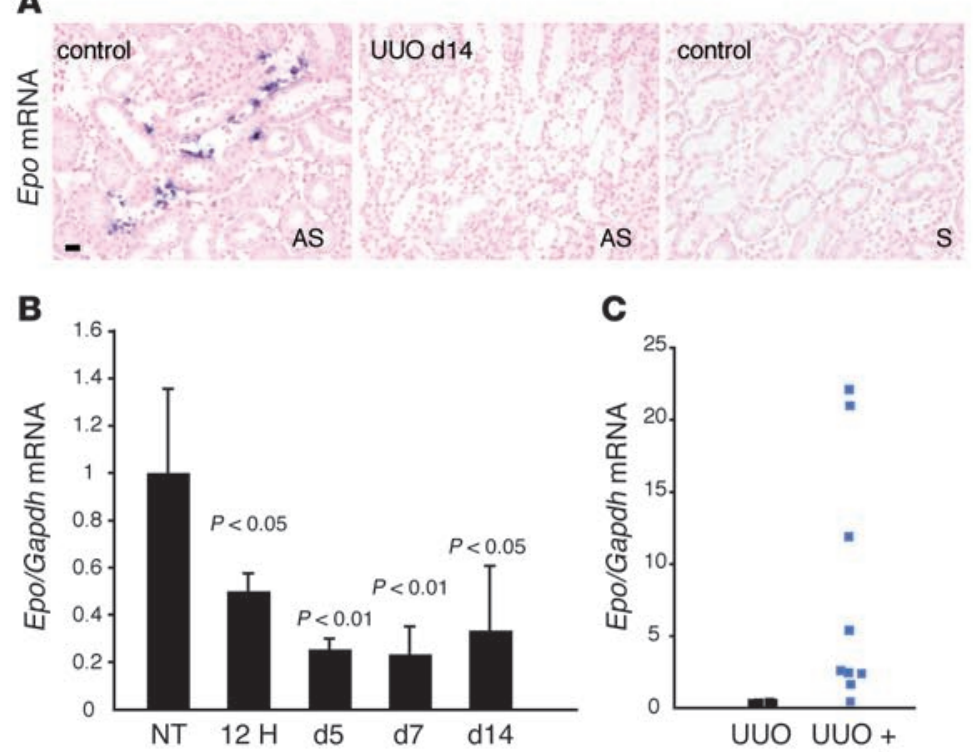

C

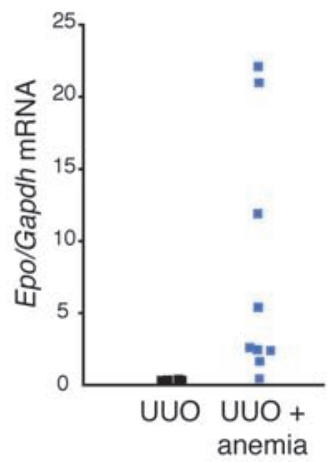

D

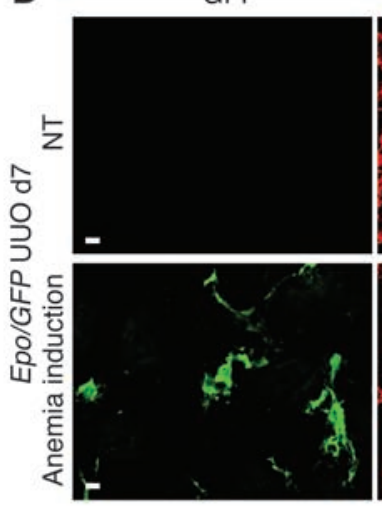

$\alpha-S M A$
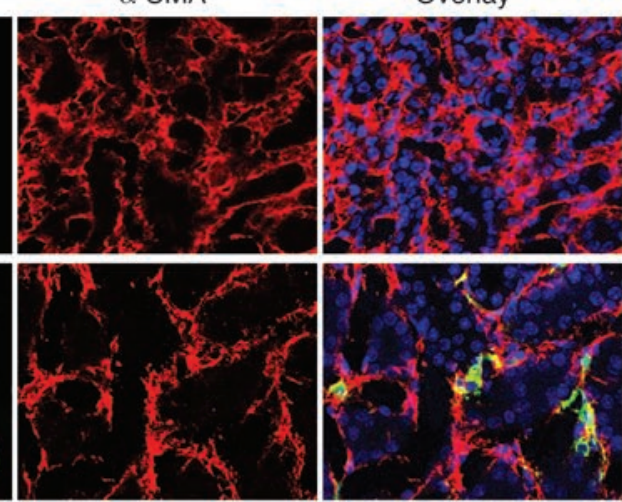

E
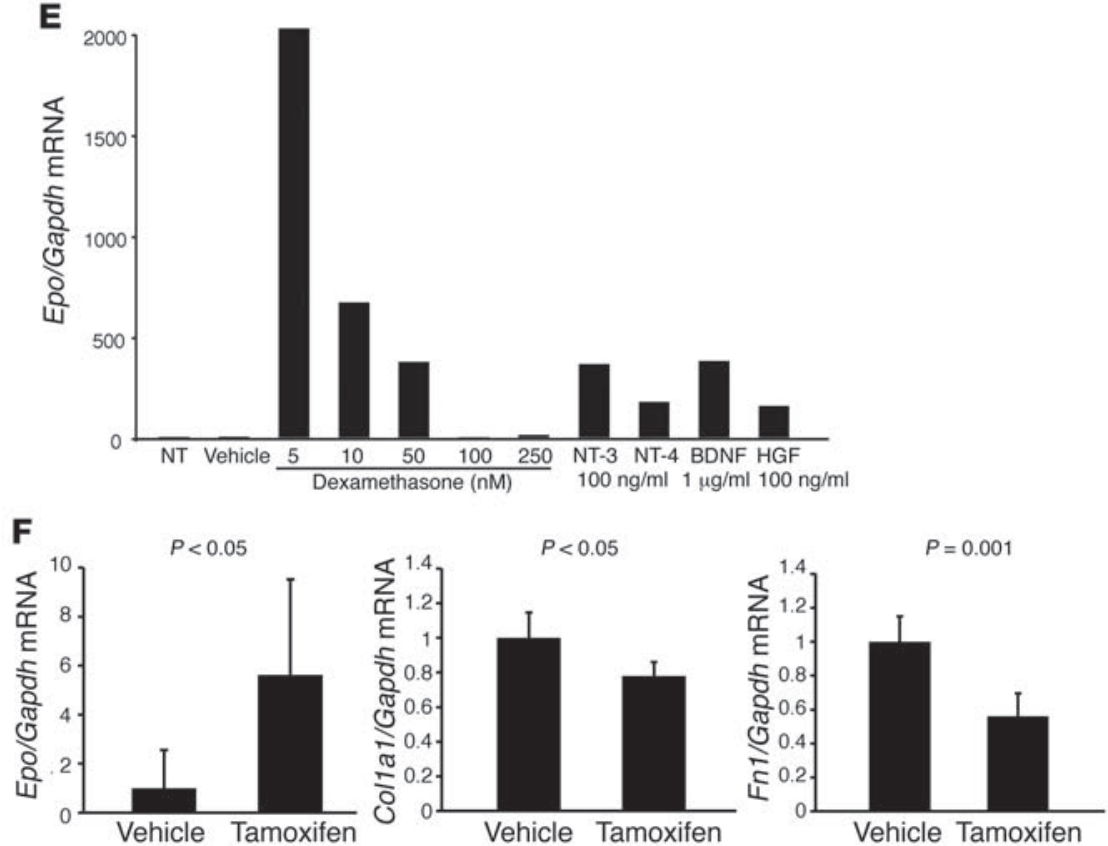

\section{Figure 5}

EPO-producing fibroblasts also transform into myofibroblasts, hindering EPO production. (A) In situ hybridization demonstrated that Epo mRNA detected in the control kidney was almost undetectable in the operated kidneys at day 14 of UUO. Hybridization with a sense probe did not elicit any signals in the control kidney. AS, antisense; S, sense. (B) Expression of Epo mRNA in the operated kidney decreased significantly, as early as 12 hours after ligation of the ureter ( $n=5 /$ group, except for the group sacrificed at day 5 [d5], in which $n=4)$. NT, nontreated. (C) The suppression of Epo mRNA in the operated kidneys was reversed after the induction of anemia. Each dot represents renal expression of Epo in an individual mouse ( $n=5$ in UUO group; $n=9$ in UUO plus anemia group). (D) Undetectable $\mathrm{GFP}^{+}$cells in the operated kidneys of Epo-GFP mice became detectable after the induction of anemia and colocalized with $\alpha$-SMA. (E) The expression of Epo in cultured myofibroblasts was augmented by the administration of lowdose dexamethasone, neurotrophins, and HGF. Vehicle used was $0.05 \%$ ethanol for the control of dexamethasone. Data are representative of results of 5 independent experiments. (F) Expression of Epo mRNA in UUO kidneys was significantly restored, whereas the expression of Col1a1 and fibronectin (Fn1) mRNA was decreased by tamoxifen treatment $(n=5)$. Vehicle used was $100 \mu \mathrm{l}$ of $10 \%$ ethanol in sunflower oil. Expression of Epo, Col1a1, and Fn1 was normalized to that of Gapdh and expressed relative to that in (B and $\mathbf{E}$ ) the nontreated group or $(\mathbf{F})$ vehicle-treated group. Scale bars: $10 \mu \mathrm{m}$.

of ECFP/Ki67 double-positive cells, indicating that the increase in the number of $\mathrm{ECFP}^{+}$cells in the operated kidney is at least partially attributable to the proliferation of $\mathrm{ECFP}^{+}$cells (Figure 4G).

Another possibility is that macrophages and dendritic cells infiltrated the operated kidneys in response to inflammation. This would account for the increase in the number of $\mathrm{ECFP}^{+}$cells in the operated kidneys. However, $\mathrm{ECFP}^{+}$cells did not overlap with the inflammatory cells, which infiltrated the operated kidneys in response to inflammation (Supplemental Figure 1, D-F). Instead, these $\mathrm{ECFP}^{+}$cells were almost exclusively positive for PDGFR- $\beta$ (Figure $4 \mathrm{H}$ ), a marker for fibroblasts, and $\alpha$-SMA (Figure $4 \mathrm{I}$ ), a marker for myofibroblasts. Quantitative analysis demonstrated that $97 \%$ of PDGFR- $\beta^{+}$interstitial cells as well as $94 \%$ of $\alpha$-SMA ${ }^{+}$myofibroblasts were positive for ECFP, whereas $97 \%$ and $93 \%$ of $\mathrm{ECFP}^{+}$cells were positive for PDGFR- $\beta$ and $\alpha$-SMA, respectively (Figure $4 \mathrm{~J}$ ). Similar results were 


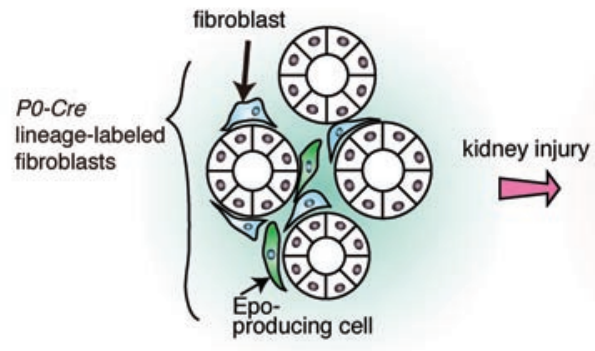

Healthy kidney
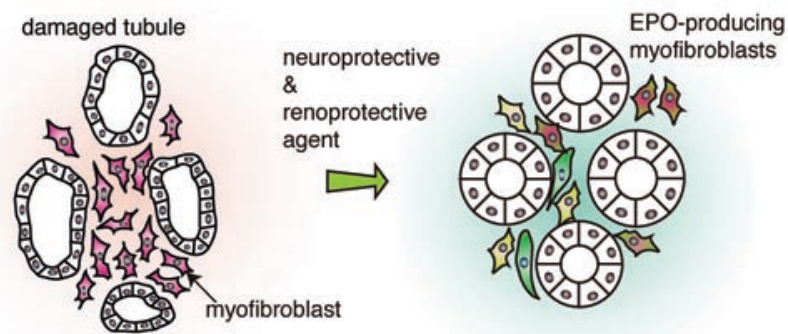

Fibrosis and Renal Anemia
Restoration of EPO Production

\section{Figure 6}

Hypothetical model for 2 different characteristics of $P O$-Cre lineage-labeled fibroblasts in the kidney, with a possible therapeutic implication for treatment of renal anemia. $P O-C r e$ lineage-labeled fibroblasts in the kidney produce EPO, which possesses hematopoietic and tissue-protective functions in healthy kidneys. In contrast, they transdifferentiate into scar-producing myofibroblasts and lose EPO-producing activity after kidney injury, leading to renal fibrosis and renal anemia. We further demonstrated that the administration of various neuroprotective and renoprotective reagents restores the EPO-producing ability in fibrotic kidneys.

obtained in other models of renal fibrosis - folic acid nephropathy (Figure $4 \mathrm{~K}$ ) and severe ischemic reperfusion injury (Figure $4 \mathrm{~L}$ ). These results indicate that $\mathrm{PO}$-Cre lineage-labeled fibroblasts are the main source of scar-producing myofibroblasts, contributing to fibrosis after kidney injury.

EPO-producing fibroblasts also transform into myofibroblasts at the cost of EPO production. Regardless of the cause of the damage, diseased kidneys invariably suffer from fibrosis with reduced EPO production. The mechanism underlying this defective production of EPO in the diseased kidney remains unknown (27). In situ hybridization detected Epo mRNA in the interstitium of the control kidney (Figure 5A, left) but not in the operated kidney at day 14 of UUO (Figure 5A, middle). Suppression of Epo mRNA in the operated kidney was evident as early as 12 hours after ligation of the ureter (Figure 5B). Interestingly, the attenuated production of Epo mRNA in the fibrotic kidney was restored by the induction of anemia (Figure 5C and Supplemental Figure 10). Similarly, GFP ${ }^{+}$ cells were almost undetectable in the fibrotic kidneys of Epo-GFP mice, whereas many $\mathrm{GFP}^{+}$cells emerged in the fibrotic kidneys after the induction of anemia (Figure 5D). These $\mathrm{GFP}^{+}$cells in the fibrotic kidneys were positive for $\alpha$-SMA (Figure 5D), whereas $\mathrm{GFP}^{+}$cells in the healthy kidneys were negative for $\alpha$-SMA (Supplemental Figure 5). These results indicate that transdifferentiated myofibroblasts still possess the capacity to produce EPO after the induction of anemia.

Taken together, the results indicate that the cause of renal anemia is not the loss of EPO-producing cells but is at least partially due to the transdifferentiation of EPO-producing cells into scar-producing myofibroblasts that express a smaller amount of EPO (Figure 6).

These findings led us to seek the possible stimuli to restore the EPO-producing ability in myofibroblasts. Because EPO-producing cells in the kidney possess both renal and neural characteristics, we administered renoprotective and neuroprotective agents to cultured primary myofibroblasts obtained from fibrotic kidneys to determine whether or not the EPO-producing ability could be restored. Among the agents that we tested, we found that the administration of neuroprotective agents, such as lowdose dexamethasone, neurotrophins (neurotrophin-3 [NT-3], NT-4, and brain-derived neurotrophic factor [BDNF]), and the renoprotective agent, HGF, increased EPO production in myofibroblasts (Figure 5E and Supplemental Figure 11). We sought other possible reagents to alleviate the loss of EPO in fibrotic kidney in vivo and found that the administration of tamoxifen, a SERM, restored the attenuated expression of EPO in fibrotic kidney (Figure 5F). SERMs activate the estrogen receptors and function as estrogen agonists in some tissues, such as bone and kidneys, and as estrogen antagonists in other tissues, such as breast. The administration of tamoxifen also restored fibrosis significantly (Figure 5F). These results indicate that the attenuated production of EPO in scar-producing myofibroblasts can be restored (Figure 6).

\section{Discussion}

In the present study, we demonstrated that EPO-producing cells in healthy kidney and scar-producing myofibroblasts during fibrosis originate from the same $\mathrm{PO}$-Cre lineage-labeled extrarenal cells, which enter the embryonic kidney at E13.5 to become renal fibroblasts and transit from one another depending on the condition of the kidney. A greater understanding of the mechanisms that control the balance between these 2 functions will lead to better treatment for patients with CKD. More importantly, the reversibility of EPO production in the fibrotic kidney (Figure 5, $\mathrm{C}-\mathrm{F})$ raised the possibility of a therapeutic approach toward renal anemia (Figure 6).

Neurotrophins are reported to promote neuronal growth and survival in many neuronal populations through the activation of Trk receptor tyrosine kinases (28-30). Furthermore, NT-3 rescues neuronal precursors from apoptosis and promotes neuronal differentiation in embryonic kidney explants (31). Given the origin of EPO-producing cells from $\mathrm{PO}$-Cre-expressing precursors, it is plausible that neurotrophins restore EPO-producing ability in transdifferentiated myofibroblasts. Recently, pharmaceutical strategies to stimulate neurotrophin signaling established proof of principle of these as effective therapeutics for Alzheimer disease (30). Among these strategies, small molecule activators of neurotrophin are appealing and should be tested in the model of renal anemia in the future.

Glucocorticoid exhibits both protective and destructive effects in the nervous system. In excess, glucocorticoid produces neuronal death, whereas low-dose glucocorticoid is neuroprotective. Recently, it has been demonstrated that glucocorticoid provides a neuroprotective effect through the activation of neurotrophin signaling 
(32), indicating that the beneficial effect of low-dose dexamethasone in the restoration of the EPO-producing ability might be exerted through neurotrophin functions as well.

On the other hand, HGF has been reported to promote the regeneration of damaged cells after kidney injury (33). The regenerative capacity of HGF may be effective in the restoration of EPOproducing ability in myofibroblasts.

Recently, Bernhardt et al. demonstrated that the pharmacological manipulation of the HIF system increases EPO production in patients with end-stage renal disease. They suggested that the disturbed oxygen sensing of EPO-producing cells causes renal anemia (34). However, the activation of HIF has multiple downstream effects, and intermittent HIF activation over a prolonged period of time may lead to profound changes in cellular metabolism, growth, and differentiation (35).

The results of the present study prompt the proposal of another possible therapeutic approach to treat renal anemia by protecting damaged EPO-producing cells. The administration of neurotrophins, low-dose dexamethasone, and HGF as well as the reagents activating these pathways may be a promising therapeutic approach either alone or in combination with other approaches. In addition, SERMs, including tamoxifen, are promising candidate drugs to treat renal anemia as well as kidney fibrosis. Recently, raloxifene, another SERM, has been reported to be renoprotective in postmenopausal women with osteoporosis (36). Clinical trials of SERMs in patients with CKD with renal anemia and further clarification of the mechanism of action of SERMs are needed.

Although we demonstrated that almost all cortical fibroblasts in the kidney arise from $\mathrm{PO}$-Cre-expressing precursors (Figure 1J), less than $20 \%$ of cortical fibroblasts produce EPO, even at the maximum induction of EPO under an anemic or hypoxic condition (27). Whether or not a certain subpopulation of $\mathrm{PO}$-Cre lineage-labeled fibroblasts is designated to possess EPO-producing ability, or all P0-Cre lineage-labeled fibroblasts are born with the ability but some environmental factors determine which cells produce EPO, remains to be clarified. Although the lack of specific cell surface markers of EPO-producing cells has hindered further analysis, we demonstrated that $\mathrm{p} 75$ is an effective surface marker in the purification of EPO-producing cells in neonatal kidney (Figure 3H). In the adult kidney, however, p75 was not an effective marker because the expression of $\mathrm{p} 75$ protein in the kidney was downregulated (data not shown), whereas its mRNA was still detected by reversetranscription PCR (RT-PCR) in EPO-producing cells (Figure 3G). A genome-wide analysis of EPO-producing cells purified from neonatal kidneys may enable the identification of suitable surface markers for the purification of the cells in adult kidneys.

We also demonstrated that more than $94 \%$ of myofibroblasts arise from $\mathrm{P} 0$-Cre lineage-labeled fibroblasts, providing what we believe to be new insights into the long-lasting controversy regarding the source of scar-producing myofibroblasts in the kidney. Recently, EMT has become widely accepted as a mechanism by which injured tubular cells transform into scar-producing cells and contribute to fibrosis during kidney injury (11). However, a number of conflicting data about the existence of EMT in vivo have been published (8, 37-39). Our data support the idea that myofibroblasts emerge from the proliferation and transdifferentiation of fibroblasts in the kidney (8), but it is not likely that they emerge from EMT of injured tubules. Recently, Humphreys et al. proposed that FoxD1+ mesenchymal cells in the developing kidneys differentiate into myofibroblasts in fibrosis (37), although the labeling efficiency of myofibroblasts by FoxD1-inducible Cre transgenic mice was $20 \%$, due to the toxicity of tamoxifen in embryogenesis. $\mathrm{PO}$-Cre fate-mapped cells populate around $\mathrm{Six}^{+}$progenitor cells in the developing kidney (Figure 2, G and $\mathrm{H}$ ), and some of them express FoxD1 (Supplemental Figure 9). From this result, we speculate that the $\mathrm{PO}$-Cre-labeled population and the FoxD1-Cre-labeled population are overlapping.

Recent reports indicate that neural crest cells contribute to bone marrow cells (22) and mesenchymal stem cells (40), in addition to the classical neural crest derivatives, such as peripheral nervous system, melanocytes, and adrenal medulla (24). The previous chickquail xenotransplantation experiment $(41,42)$ as well as the dye injection into the dorsal neural tube (43) indicate that neural crestderived cells contribute to the developing kidneys (44). This observation is also supported by the results in this manuscript, showing that most fibroblasts in the kidney arose from $\mathrm{PO}$-Cre-expressing precursors (Figure 1, H-J) and that neonatal kidney fibroblasts expressed neural crest marker p75 (Figure $1 \mathrm{~K}$ ). On the contrary, a previous lineage-tracing study using Wnt1-Cre mice (45), another tool to trace the fate of neural crest derivatives, does not reveal a pattern overlapping with that of $\mathrm{PO}$-Cre mice in the kidney. One possible explanation for the discordant results might be a difference in the efficiency and timing of labeling neural crest-derived cells in these Cre drivers. For instance, in Wnt1-Cre mice, Cre is predominantly expressed in the dorsal neural tube from which neural crest cells delaminate, whereas in $\mathrm{PO}$-Cre mice, Cre expression is sustained in migrating neural crest cells. Although there is a possibility that the $\mathrm{P} 0$-Cre driver is ectopically expressed in other precursor tissues, $\mathrm{PO}$-Cre-driven lineage labeling has to occur outside of the embryonic kidney, since we did not detect Cre expression within the developing kidney (Supplemental Figures 3 and 8).

Although further study is needed before one can definitely conclude that $\mathrm{PO}$-Cre lineage-labeled cells are of neural crest origin, the hypothesis that EPO-producing cells derive from neural crest cells might open a new avenue of research toward the in vitro generation of EPO-producing cells from patient-derived induced pluripotent stem (iPS) cells. This is promising because an efficient method for converting iPS cells to neural crest cells has already been established in a recent study (46).

\section{Methods}

Animals. Transgenic mice expressing Cre under the control of the P0 promoter (P0-Cre mice) (17) were mated with floxed-EGFP mice (CAG-CAT ${ }^{\text {loxP/loxP }}$ EGFP mice; ref. 18) (a gift from J. Miyazaki of Osaka University, Osaka, Japan), R26R mice (19), R26ECFP mice (20) (a gift from F. Costantini of Colombia University, New York, New York, USA), and R26tdRFP mice (21) to obtain double-transgenic mice. Epo-GFP mice (6) were described previously. Adult wild-type mice were purchased from Japan SLC. Anemia was induced by bleeding (6) as well as by the administration of phenylhydrazine as described previously (47). The GFP signal in Epo-GFP mice was observed after the induction of anemia. All animal studies were approved by the Animal Research Committee, Graduate School of Medicine, Kyoto University, and were conducted in accordance with the Guide for the Care and Use of Laboratory Animals (US NIH).

Kidney disease models. UUO and folic acid nephrotoxicity was induced as described previously $(48,49)$. Severe ischemic reperfusion injury was induced by 75 -minute clamp of the unilateral renal pedicles: the kidneys were harvested 3 weeks after the operation. Tamoxifen $(5 \mathrm{mg} / 20 \mathrm{~g} \mathrm{BW})$ was orally administered everyday from 5 days before UUO to the day of operation and every 2 days after the operation. 
Renal histopathology. Immunostaining was performed as described previously (50) using the following primary antibodies: anti-GFP (Molecular Probes), p75 (Advanced Targeting Systems), PDGFR- $\beta$ (eBioscience), Six 2 (Proteintech Group), $\alpha$-SMA (Sigma-Aldrich), TUJ1-FITC (Covance), TH (Chemicon), Cre (Novagen), P0 (Novus Biologicals), LacZ (Cappel), RFP (Abcam), FoxD1 (a gift from A.P. McMahon and J.W. Mugford of Harvard University, Cambridge, Massachusetts, USA) (51), and CD11b, CD11c, CD31, CD73 (all from Pharmingen). EGFP and ECFP were visualized by immunostaining with anti-GFP antibody. tdRFP was visualized by immunostaining with anti-RFP antibody.

Flow cytometric analysis. Flow cytometric analysis was performed using BD FACSAria. Mice were anesthetized and then perfused with normal saline. Kidneys were harvested, and the tissue was minced with a razor blade and digested by $1 \mathrm{mg} / \mathrm{ml}$ collagenase A and $0.1 \mathrm{mg} / \mathrm{ml}$ DNase type I as previously described (52). The cell suspensions were filtered through a cell strainer to remove debris and were stained with antibodies against GFP (Molecular Probes) and p75 (Advanced Targeting Systems). The cells were fixed in $4 \%$ PFA for GFP staining but not for p 75 staining.

Myofibroblast culture. UUO kidneys were harvested 14 days after the operation in the same manner used in the preparation of cell suspension for FACS analysis. Cells from 1 UUO kidney were seeded onto a Primaria 6-well dish (BD Bioscience), washed twice, stimulated with various agents 24 hours later, and incubated 48 hours after the stimulation in a hypoxic chamber $\left(1 \% \mathrm{O}_{2}\right)$. We confirmed that most, if not all, $\alpha-\mathrm{SMA}^{+}$cultured myofibroblasts from P0-Cre/R26ECFP mice were positive for ECFP (Supplemental Figure 6). We also confirmed the presence of EPO protein $(0.2-0.75 \mathrm{pg} / \mathrm{ml})$ in the culture supernatant of the cells using ELISA (Mouse/Rat EPO Quantikine ELISA system, R\&D Systems).

Statistics. All assays were performed at least 5 times. Data are presented as the mean \pm SD. Statistical significance was assessed by Student's $t$ test for 2 group comparisons. $P<0.05$ is considered significant.

\section{Acknowledgments}

We thank M. Yanagisawa, M. Nakafuku, R. Kageyama, T. Ohtsuka, Y. Kaziro, T. Nakamura, Y. Nabeshima, S. Nagata, K. Ohta, and A. Economides for their valuable comments and discussion. We also greatly appreciate F. Costantini for the R26ECFP mice, J. Miyazaki for the CAG-CAT ToxP/loxP-EGFP mice, T. Yamamoto for his kind help with in situ hybridization, T. Kurosaki and H. Kawamoto for the valuable help in the transfer of the R26tdRFP mice, and A.P. McMahon and J.W. Mugford for the FoxD1 antibodies and protocol for immunostaining. We sincerely thank C. Gerle for the critical reading of this manuscript. We are very grateful to A. Hosotani for her excellent technical assistance. This study was supported by the Funding Program for Next-Generation World-leading Researchers; Grantsin-Aid from the Ministry of Education, Culture, Science, Sports and Technology of Japan (Shin-gakujyutsu 21200080); a grant-in-aid for Research on Biological Markers for New Drug Development; Health and Labor Sciences Research Grants from the Ministry of Health, Labor and Welfare of Japan (08062855); grants from Astellas Foundation for Research on Metabolic Disorders; Novartis Foundation for the Promotion of Science; Senri Lifescience Foundation; Mochida Memorial Foundation; Takeda Science Foundation; and Japan Foundation for Applied Enzymology.

Received for publication February 25, 2011, and accepted in revised form July 26, 2011.

Address correspondence to: Motoko Yanagita, Career-Path Promotion Unit for Young Life Scientists, E-wing, Kyoto University Graduate School of Medicine, Yoshida-konoe-cho, Sakyo-ku, Kyoto 6068501, Japan. Phone: 81.75.753.9310; Fax: 81.75.753.9311; E-mail: motoy@kuhp.kyoto-u.ac.jp.
1. Fisher JW. A quest for erythropoietin over nine decades. Annu Rev Pharmacol Toxicol. 1998;38:1-20.

2. Erslev AJ, Besarab A. Erythropoietin in the pathogenesis and treatment of the anemia of chronic renal failure. Kidney Int. 1997;51(3):622-630.

3. Soni RK, Weisbord SD, Unruh ML. Health-related quality of life outcomes in chronic kidney disease. Curr Opin Nephrol Hypertens. 2010;19(2):153-159.

4. Noguchi CT, Wang L, Rogers HM, Teng R, Jia Y. Survival and proliferative roles of erythropoietin beyond the erythroid lineage. Expert Rev Mol Med. 2008; 10:e36.

5. Bachmann S, Le Hir M, Eckardt KU. Co-localization of erythropoietin mRNA and ecto-5'-nucleotidase immunoreactivity in peritubular cells of rat renal cortex indicates that fibroblasts produce erythropoietin. J Histochem Cytochem. 1993;41(3):335-341.

6. Obara N, Suzuki N, Kim K, Nagasawa T, Imagawa $\mathrm{S}$, Yamamoto M. Repression via the GATA box is essential for tissue-specific erythropoietin gene expression. Blood. 2008;111(10):5223-5232.

7. Bhowmick NA, Neilson EG, Moses HL. Stromal fibroblasts in cancer initiation and progression. Nature. 2004;432(7015):332-337.

8. Kriz W, Kaissling B, Le Hir M. Epithelialmesenchymal transition (EMT) in kidney fibrosis: fact or fantasy? J Clin Invest. 2011;121(2):468-474.

9. Kaissling B, Le Hir M. The renal cortical interstitium: morphological and functional aspects. Histochem Cell Biol. 2008;130(2):247-262.

10. Wada T, Sakai N, Matsushima K, Kaneko S. Fibrocytes: a new insight into kidney fibrosis. Kidney Int. 2007;72(3):269-273.

11. Iwano M, Plieth D, Danoff TM, Xue C, Okada H, Neilson EG. Evidence that fibroblasts derive from epithelium during tissue fibrosis. J Clin Invest. 2002; 110(3):341-350.
12. Duffield JS, Bonventre JV. Kidney tubular epithelium is restored without replacement with bone marrow-derived cells during repair after ischemic injury. Kidney Int. 2005;68(5):1956-1961.

13. Lin F, Moran A, Igarashi P. Intrarenal cells, not bone marrow-derived cells, are the major source for regeneration in postischemic kidney. J Clin Invest. 2005 . 115(7):1756-1764.

14. Roufosse $C$, et al. Bone marrow-derived cells do not contribute significantly to collagen I synthesis in a murine model of renal fibrosis. J Am Soc Nephrol. 2006;17(3):775-782.

15. Lin SL, Kisseleva T, Brenner DA, Duffield JS. Pericytes and perivascular fibroblasts are the primary source of collagen-producing cells in obstructive fibrosis of the kidney. Am J Pathol. 2008; 173(6):1617-1627.

16. Humphreys BD, et al. Intrinsic epithelial cells repair the kidney after injury. Cell Stem Cell. 2008; 2(3):284-291

17. Yamauchi $Y$, et al. A novel transgenic technique that allows specific marking of the neural crest cell lineage in mice. Dev Biol. 1999;212(1):191-203.

18. Kawamoto S, et al. A novel reporter mouse strain that expresses enhanced green fluorescent protein upon Cre-mediated recombination. FEBS Lett. 2000; 470(3):263-268.

19. Soriano P. Generalized lacZ expression with the ROSA26 Cre reporter strain. Nat Genet. 1999; 21(1):70-71.

20. Srinivas $S$, et al. Cre reporter strains produced by targeted insertion of EYFP and ECFP into the ROSA26 locus. BMC Dev Biol. 2001;1:4.

21. Luche H, Weber O, Nageswara Rao T, Blum C, Fehling HJ. Faithful activation of an extra-bright red fluorescent protein in "knock-in" Cre-reporter mice ideally suited for lineage tracing studies. EurJ
Immunol. 2007;37(1):43-53.

22. Nagoshi N, et al. Ontogeny and multipotency of neural crest-derived stem cells in mouse bone marrow, dorsal root ganglia, and whisker pad. Cell Stem Cell. 2008;2(4):392-403.

23. Andrae J, Gallini R, Betsholtz C. Role of plateletderived growth factors in physiology and medicine. Genes Dev. 2008;22(10):1276-1312.

24. Nagoshi N, Shibata S, Nakamura M, Matsuzaki Y, Toyama Y, Okano H. Neural crest-derived stem cells display a wide variety of characteristics. J Cell Biochem. 2009;107(6):1046-1052.

25. Kobayashi A, et al. Six2 defines and regulates a multipotent self-renewing nephron progenitor population throughout mammalian kidney development. Cell Stem Cell. 2008;3(2):169-181.

26. Grande MT, Lopez-Novoa JM. Fibroblast activation and myofibroblast generation in obstructive nephropathy. Nat Rev Nephrol. 2009;5(6):319-328.

27. Nangaku M, Eckardt KU. Pathogenesis of renal anemia. Semin Nephrol. 2006;26(4):261-268.

28. Chao MV. Neurotrophins and their receptors: a convergence point for many signalling pathways. Nat Rev Neurosci. 2003;4(4):299-309.

29. Patapoutian A, Reichardt LF. Trk receptors: mediators of neurotrophin action. Curr Opin Neurobiol. 2001;11(3):272-280.

30. Webster NJ, Pirrung MC. Small molecule activators of the Trk receptors for neuroprotection. BMC Neurosci. 2008;9 suppl 2:S1.

31. Karavanov A, Sainio K, Palgi J, Saarma M, Saxen L, Sariola H. Neurotrophin 3 rescues neuronal precursors from apoptosis and promotes neuronal differentiation in the embryonic metanephric kidney. Proc Natl Acad Sci US A. 1995;92(24):11279-11283.

32. Jeanneteau F, Garabedian MJ, Chao MV. Activation of Trk neurotrophin receptors by glucocorticoids 
provides a neuroprotective effect. Proc Natl Acad Sci US A. 2008;105(12):4862-4867.

33. Liu Y. Hepatocyte growth factor in kidney fibrosis: therapeutic potential and mechanisms of action. Am J Physiol Renal Physiol. 2004;287(1):F7-F16.

34. Bernhardt WM, et al. Inhibition of prolyl hydroxylases increases erythropoietin production in ESRD. J Am Soc Nephrol. 2010;21(12):2151-2156.

35 . Haase VH. Hypoxic regulation of erythropoiesis and iron metabolism. Am J Physiol Renal Physiol. 2010; 299(1):F1-F13

36. Melamed ML, et al. Raloxifene, a selective estrogen receptor modulator, is renoprotective: a post-hoc analysis. Kidney Int. 2011;79(2):241-249.

37. Humphreys BD, et al. Fate tracing reveals the pericyte and not epithelial origin of myofibroblasts in kidney fibrosis. Am J Pathol. 2010;176(1):85-97.

38. Li L, Zepeda-Orozco D, Black R, Lin F. Autophagy is a component of epithelial cell fate in obstructive uropathy. Am J Pathol. 2010;176(4):1767-1778.

39. Koesters R, et al. Tubular overexpression of transforming growth factor-beta 1 induces autophagy and fibrosis but not mesenchymal transition of renal epithelial cells. Am J Pathol. 2010;177(2):632-643.

40. Morikawa S, et al. Development of mesenchymal stem cells partially originate from the neural crest. Biochem
Biophys Res Commun. 2009;379(4):1114-1119.

41. Le Douarin NM, Teillet MA. Experimental analysis of the migration and differentiation of neuroblasts of the autonomic nervous system and of neurectodermal mesenchymal derivatives, using a biological cell marking technique. Dev Biol. 1974;41(1):162-184.

42. Le Douarin NM. The avian embryo as a model to study the development of the neural crest: a long and still ongoing story. Mech Dev. 2004;121(9):1089-1102.

43. Bronner-Fraser M, Fraser SE. Cell lineage analysis reveals multipotency of some avian neural crest cells. Nature. 1988;335(6186):161-164.

44. Sainio K, Nonclercq D, Saarma M, Palgi J, Saxen L, Sariola H. Neuronal characteristics in embryonic renal stroma. Int J Dev Biol. 1994;38(1):77-84.

45. Itaranta P, Viiri K, Kaartinen V, Vainio S. Lumbosacral neural crest derivatives fate mapped with the aid of Wnt-1 promoter integrate but are not essential to kidney development. Differentiation. 2009; 77(2):199-208

46. Chambers SM, Fasano CA, Papapetrou EP, Tomishima M, Sadelain M, Studer L. Highly efficient neural conversion of human ES and iPS cells by dual inhibition of SMAD signaling. Nat Biotechnol. 2009;27(3):275-280.

47. Menon MP, Karur V, Bogacheva O, Bogachev O,
Cuetara B, Wojchowski DM. Signals for stress erythropoiesis are integrated via an erythropoietin receptor-phosphotyrosine-343-Stat5 axis. J Clin Invest. 2006;116(3):683-694.

48. Yanagita $M$, et al. Uterine sensitization-associated gene-1 (USAG-1), a novel BMP antagonist expressed in the kidney, accelerates tubular injury. J Clin Invest. 2006;116(1):70-79.

49. Tanaka M, et al. Expression of BMP-7 and USAG-1 (a BMP antagonist) in kidney development and injury. Kidney Int. 2008;73(2):181-191.

50. Tanaka $M$, et al. Loss of the BMP antagonist USAG-1 ameliorates disease in a mouse model of the progressive hereditary kidney disease Alport syndrome. J Clin Invest. 2010;120(3):768-777.

51. Mugford JW, Sipila P, McMahon JA, McMahon AP. Osr1 expression demarcates a multi-potent population of intermediate mesoderm that undergoes progressive restriction to an Osr1-dependent nephron progenitor compartment within the mammalian kidney. Dev Biol. 2008;324(1):88-98.

52. Vielhauer V, Anders HJ, Perez de Lema G, Luckow $\mathrm{B}$, Schlondorff D, Mack M. Phenotyping renal leukocyte subsets by four-color flow cytometry: characterization of chemokine receptor expression. Nephron Exp Nephrol. 2003;93(2):e63. 